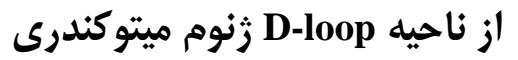

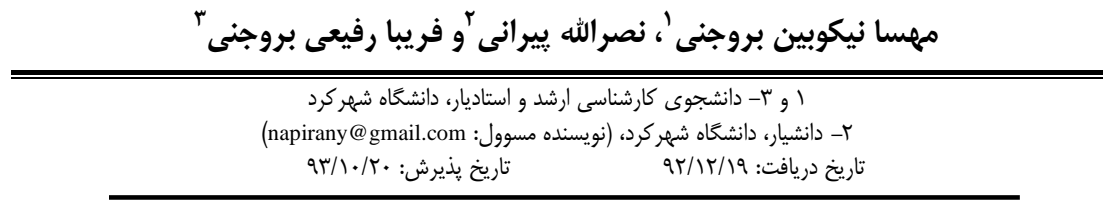

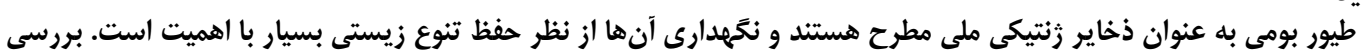

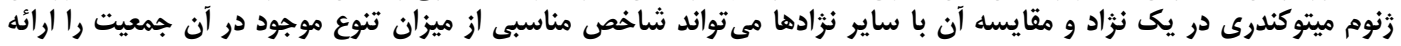

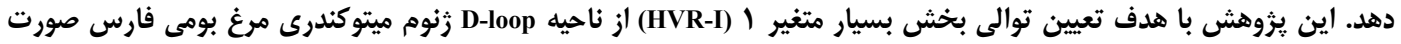

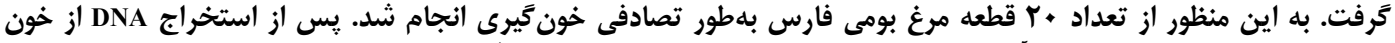

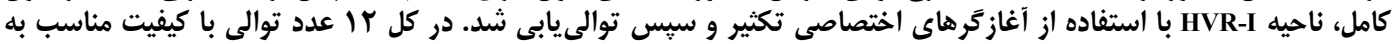

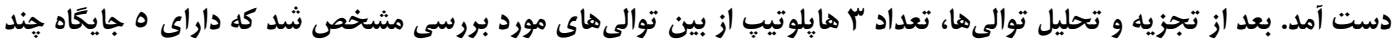

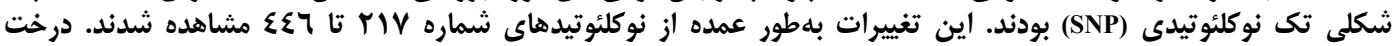

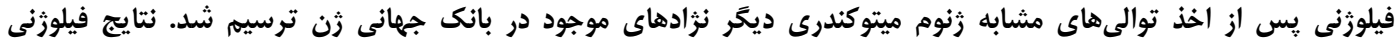

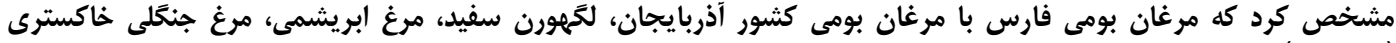

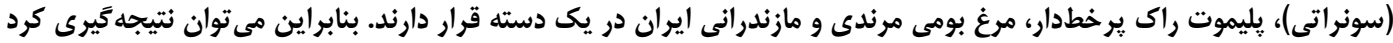

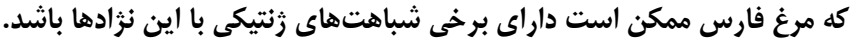

وازههاى كليدى: زنوم ميتوكندرى، ناحيه بسيار متغير (HVR-I))، جندشكلى تك نوكلئوتيدى (SNP)، فيلوزنى

ناحيه كنترل ب (HVR-III) و قطعه ميانى يا ناحيه كنترل

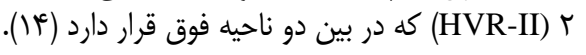

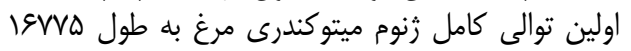

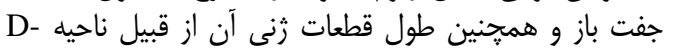

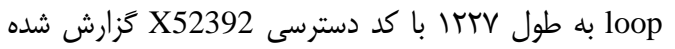

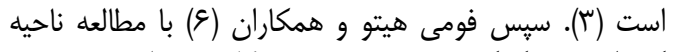
كنترل غير كدكننده

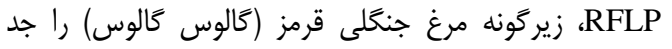

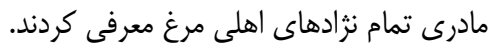

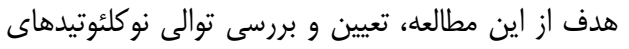

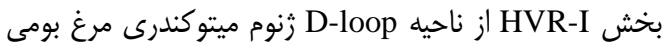

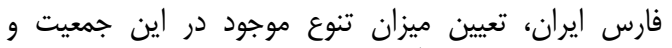

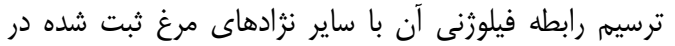
بانك جهانى ثن بود.

\section{مواد و روشها}

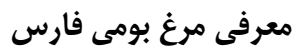

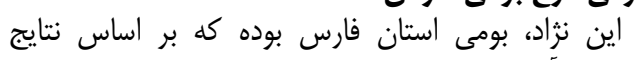

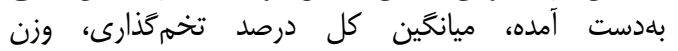

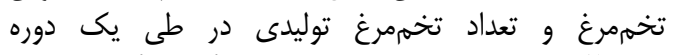
تخمثذارى 年

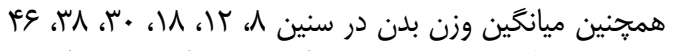

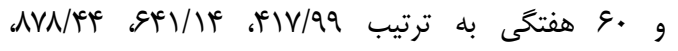
شذه

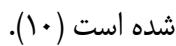

نزادهاى بومى، از ذخاير مهم زنتيكى به شمار مىروند و از

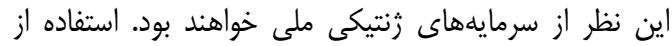

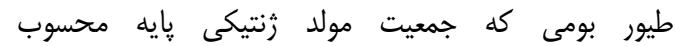

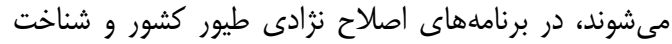

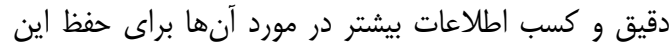

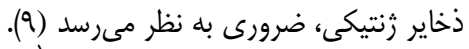

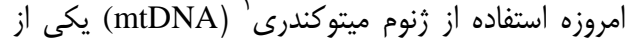

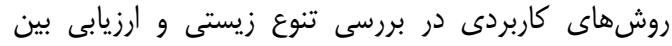

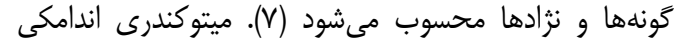

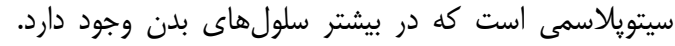

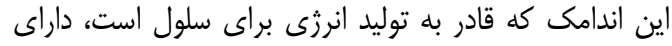

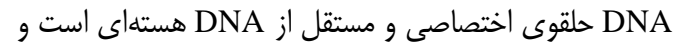

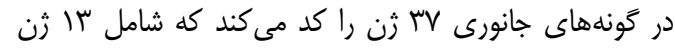

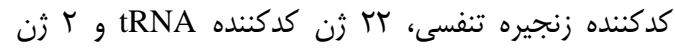

كدكننده rRNA است (1) (1).

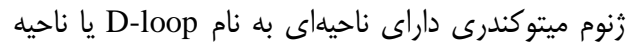

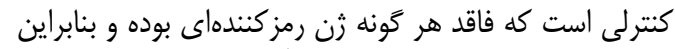

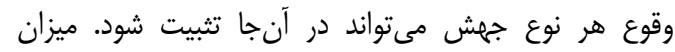

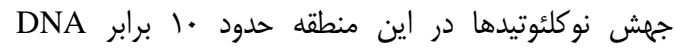

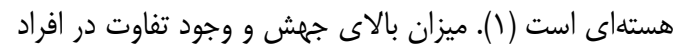

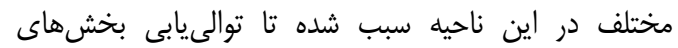

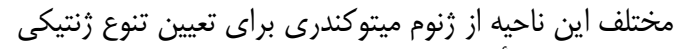

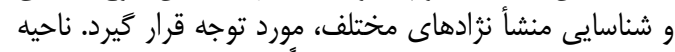

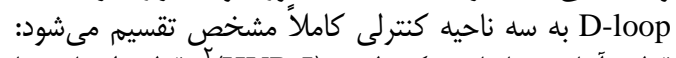

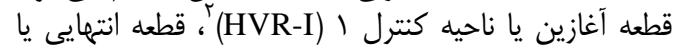


USB

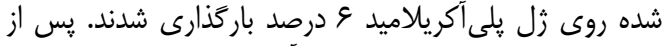

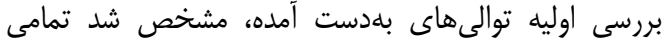

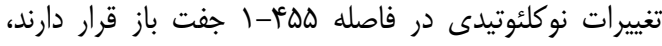

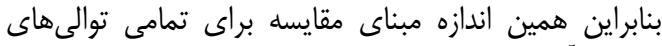

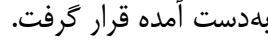
تجزيه و تحليل توالىها

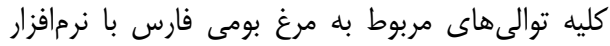

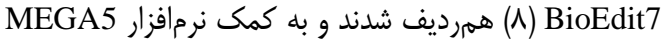

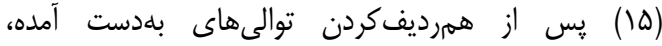

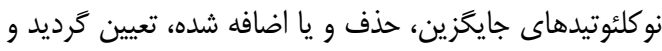

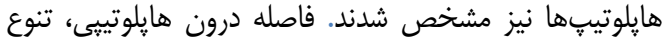

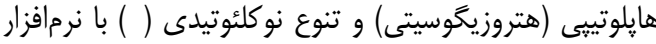

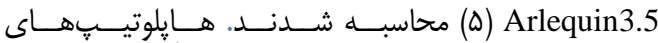

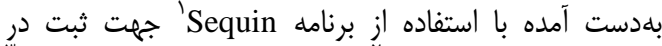

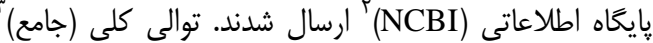

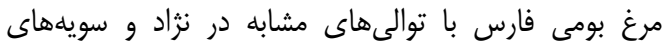
مختلف مرغ اهلى اخذ شده از بانكى ثن، در ابتدا با نرمافزار MEGA5

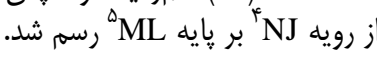

\section{نتايج و بحث}

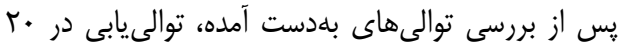

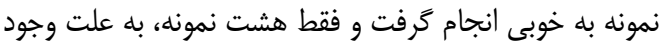

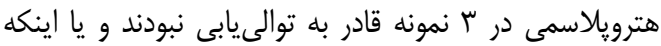

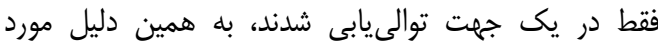

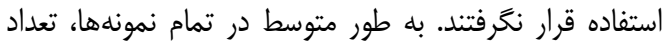

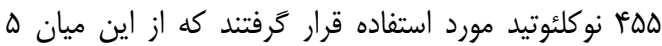

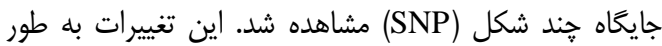

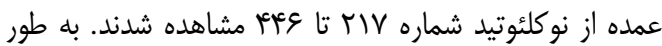

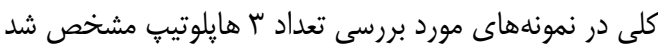

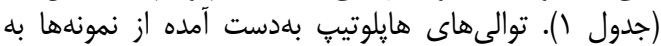

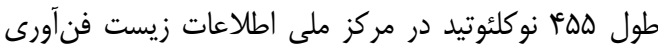
(NCBI)

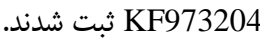

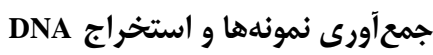

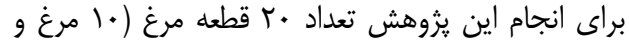

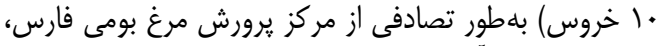

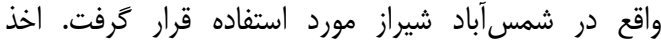

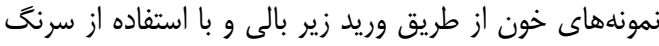

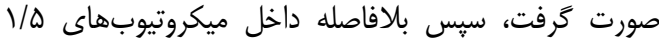

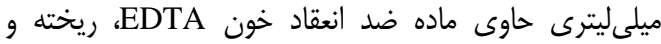

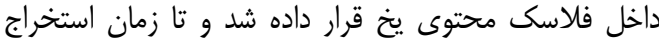

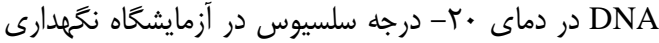

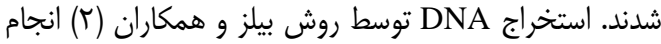

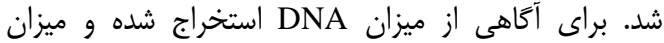

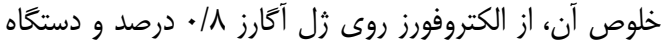

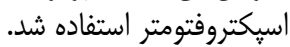

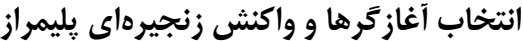

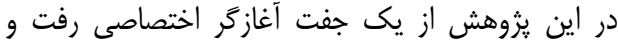

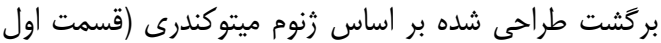

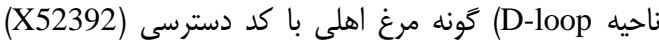

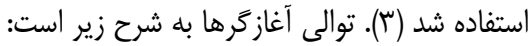

F (5'-GGCTTGAAAAGCCATTGTTG-3)

R (5'-CCCCAAAAAGAGAAGGAACC-3)

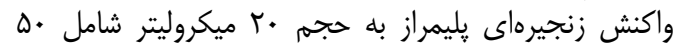

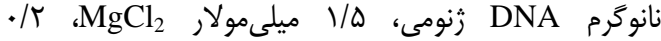

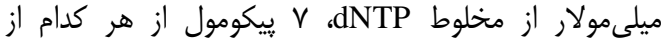

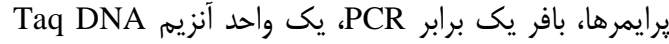

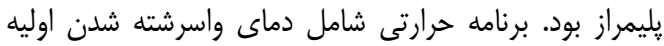

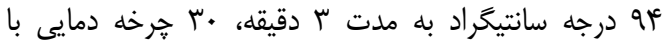

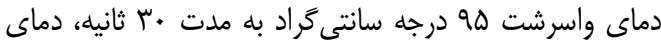

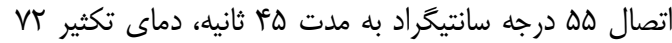

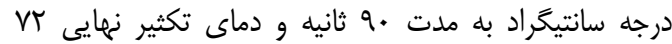

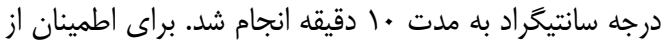

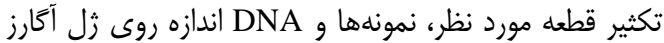

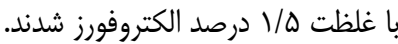

تعيين توالى محصولات تكثير شاتده

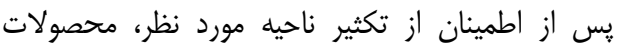

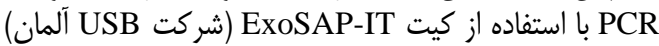
تخليص شده و سيس با استفاده إه از كيت توالىيابى (شركت

جدول ا- SNP و هايلوتيٍٍاى بلدست آمده براى نمونهاى مرغ بومى فارس Table 1. SNP and Haplotypes of Fars native chicken

\begin{tabular}{|c|c|c|c|c|c|c|}
\hline \multicolumn{5}{|c|}{ موقعيت SNP } & \multirow[t]{2}{*}{ هايلوتيب } & \multirow[t]{2}{*}{ فراوانى } \\
\hline TIV & TFE & TAI & $r . q$ & fis & & \\
\hline $\mathrm{C}$ & $\mathrm{C}$ & A & $\mathrm{T}$ & $\mathrm{T}$ & 1 & 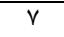 \\
\hline . & $\mathrm{T}$ & $\cdot$ & . & . & r & 1 \\
\hline $\mathrm{T}$ & . & G & $\mathrm{C}$ & $\mathrm{C}$ & r & f \\
\hline
\end{tabular}

1- http://www.ncbi.nlm.nih.gov/Sequin/ 4- Neighbor-Joining
2- National Center for Biotechnology Information 5- Maximum Likelihood
3- Consensus 


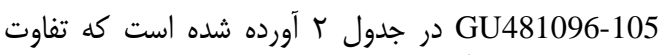

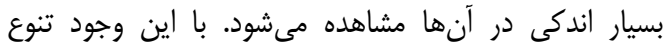

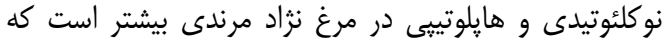
بيان كر وجود تنوع بيشتر در اين جمعيت مى باشئ.

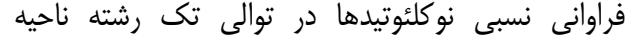

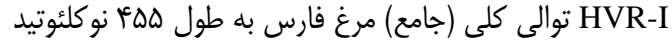

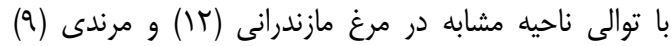

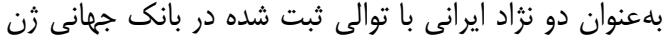

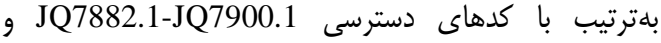

جدول r- تعداد هايلوتيب، درصد فراوانى نسبى نوكلئوتيدها، تنوع نوكلئوتيدى و هايلوتيبى ناحيه HVR-I مرغ فارس، مرندى (9) و مازندرانى (T)

Table 2. No of haploteps, percentage of relative frequencies, nucleotide and halpotype diversity in HVR-I region of Fars, Marandi (9) and Mazandran (12) native chickens

\begin{tabular}{|c|c|c|c|c|c|c|c|c|}
\hline تنوع هايلوتيبى & تنوع نوكلئوتيدى & $\mathrm{T}$ & $\mathrm{C}$ & G & A & تعداد هايلوتيب & ت تعداد نمونه & جمعيت \\
\hline$\cdot / \Delta 9 \pm \cdot / 1 \cdot V 9$ & سוץ.•/• & $r \cdot / r$ & $T V / T$ & $\mid r / 1$ & $r q / r$ & $r$ & IT & فارس \\
\hline $.14 r \pm . / .999$ & $. / .110 \pm . .1 . .9 \wedge 9$ & $r \cdot / r$ & $T V / \Delta$ & $\mid r / 1$ & $r q / r$ & $\Delta$ & 1. & 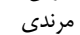 \\
\hline$\cdot / V \gamma^{c} \pm \cdot / \cdot \wedge \Delta$ & $\cdot / \cdot \Delta \mid \pm \cdot / \cdot$ r TYF & $r \cdot / r$ & $T V / S$ & $15 / 1$ & $r q / r$ & 9 & 19 & مازندرانى \\
\hline
\end{tabular}

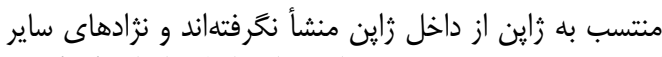

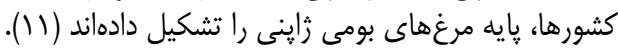

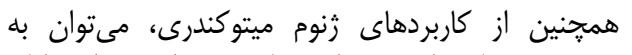

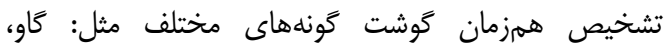

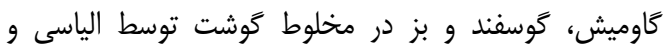
همكاران (أ) اشاره كرد.

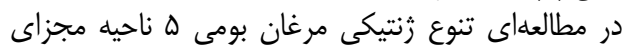

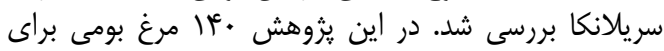

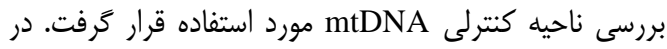

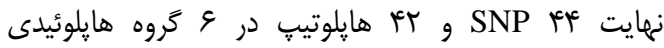

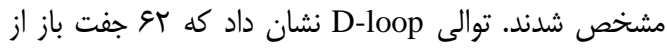

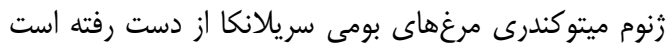

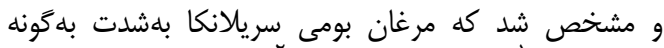

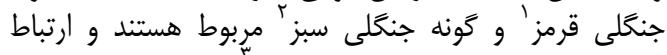

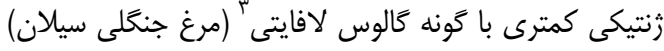

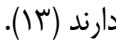

شكل I نمودار فيلوزنى رسم شده توالى كلى

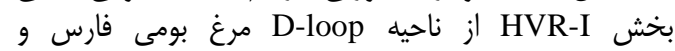

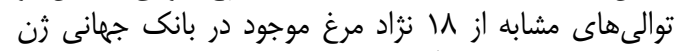

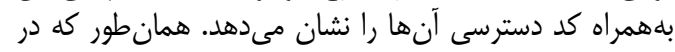

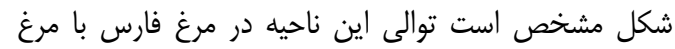

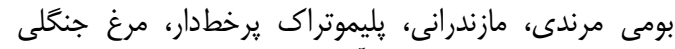

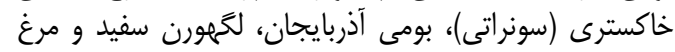

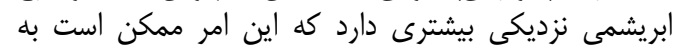

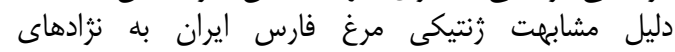

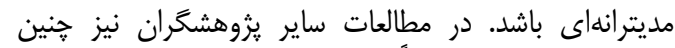

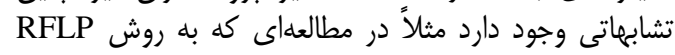

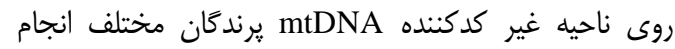

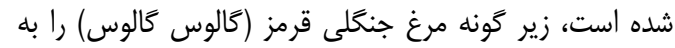

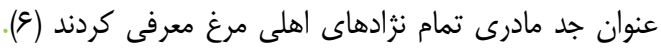

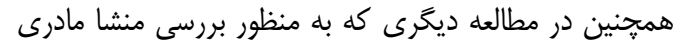

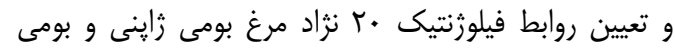
اندونزيايى انجام دادند نتيجه كرفتند كه بعزي بعضى نزادهاى 


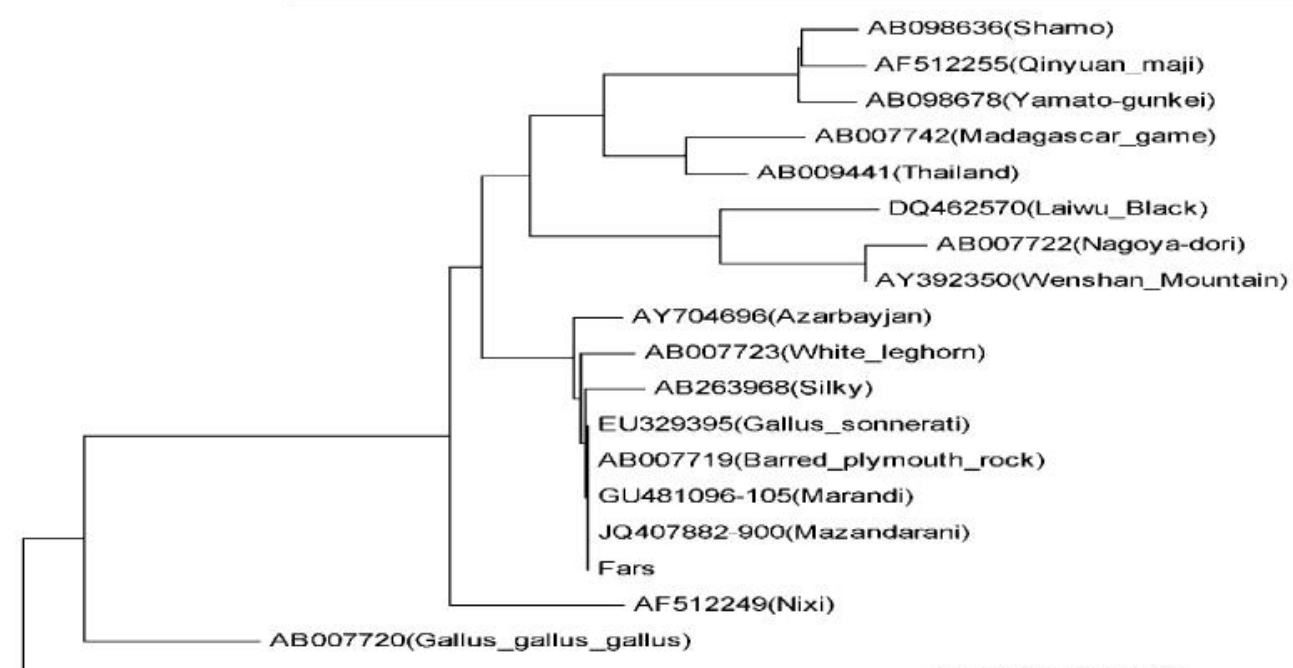

AB007718(Bankiva)

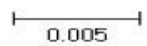

شكل ا- نمودار فيلوزنى (نمودار درختى N-J) توالى كلى (جامع) ناحيه تكثير شده از زنوم ميتوكندرى مرغ بومى فارس و برخى از نزادهاى مرغ

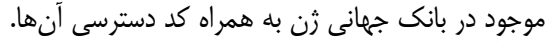

Figure 1. Phylogentic tree (N-J tree) of mitochondrial consensus sequence of Fars native chicken and other chickens available in Genbank with their accession numbers

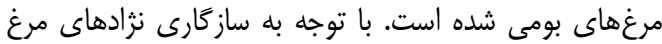

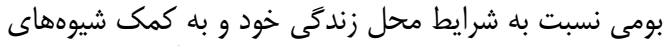

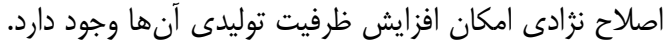

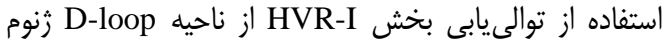

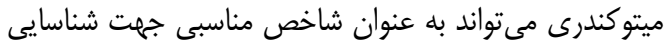

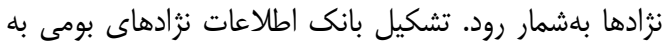

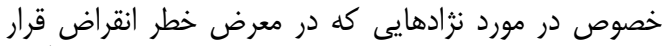

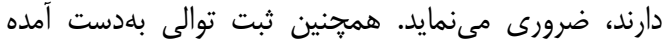

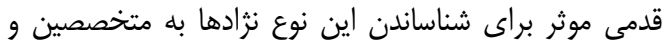

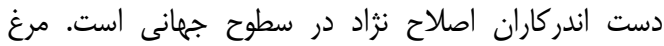

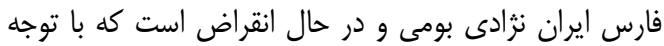

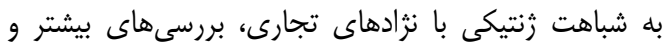

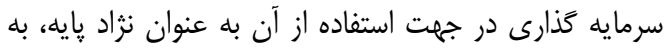

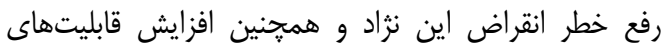

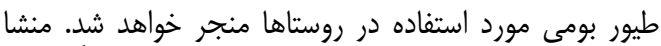

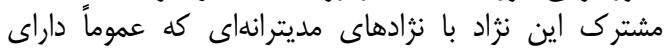

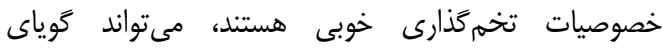

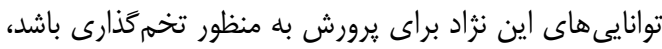

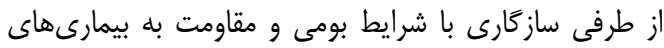
منطقهاى نيز از مزاياى اين نزاد إداد است.

$$
\begin{aligned}
& \text { تشكر و قدردانى }
\end{aligned}
$$

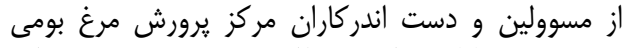

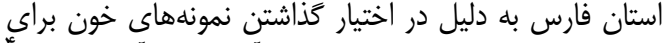

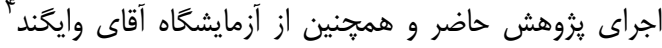

$$
\begin{aligned}
& \text { به ياس كمكىهاى ارزندهشان در تعيين توالى نمونه إنها تشكر و } \\
& \text { قدردانى مىشود. }
\end{aligned}
$$

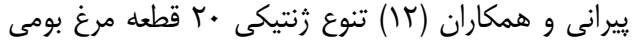

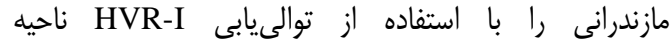

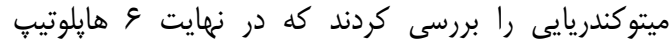

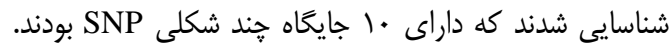

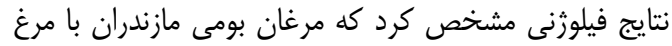

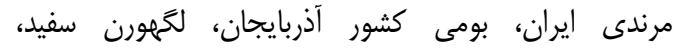

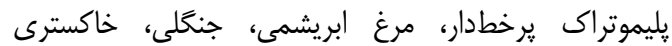

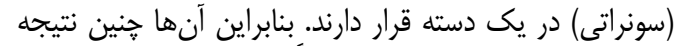

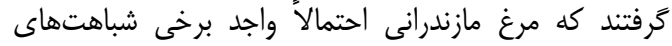
زنتيكى با اين نزادها مىباشد.

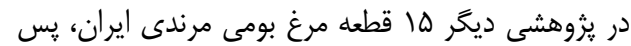

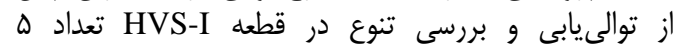

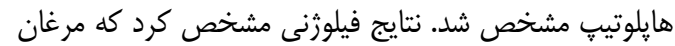

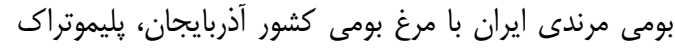

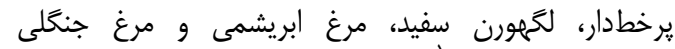

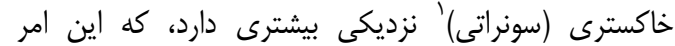

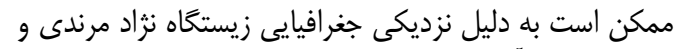

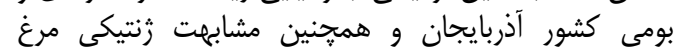

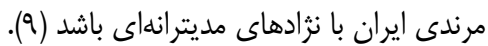

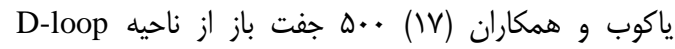

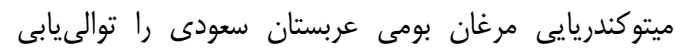

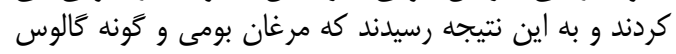

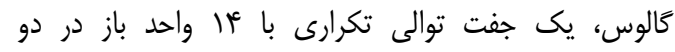

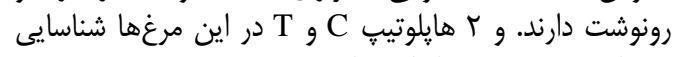

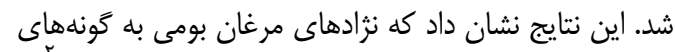

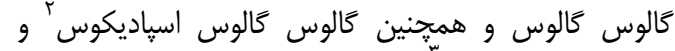

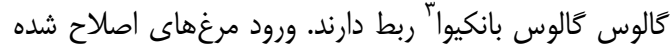

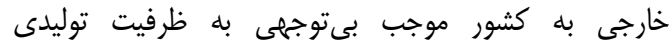

1- Gallus gallus Sonerati 2- Gallus gallus Spadiceus 4- S.Weigend (Institute of Farm Animal Genetics, Mariensee, Germany) 
1. Anderson, S.A.T., G. Bankier, M. Barrell, A. De Bruijn, J. Couson, I. Drouin, B. Nierlich, F. Roe, PH. Sanger and Young I.G. 1981. Sequence and organization of the human mitochondrial genome. Nature, 290: 457-465.

2. Bailes, S.M., J.J. Devers, J.D. Kirby and D.D. Rhoads. 2007. An expensive, simple protocol for DNA isolation from blood for high-throughput genotyping by polymerase chain reaction or restriction endonuclease digestion. Poultry Science, 86: 102-106.

3. Desjardins, P. and R. Morais. 1990. Sequence and gene organization of the chicken mitochondrial genome. A novel gene order in higher vertebrates. Journal of Molecular Biology, 212: 599-634.

4. Elyasi Zarringhabaie, G.H., N. Pirany and A. Javanmard. 2011. Molecular traceability of the species origin of meats using multiplex PCR. African Journal of Biotechnology, 10: 15461-16465.

5. Excoffier, L., G. Laval and S. Schneider. 2005. Arlequin version 3.0: an integrated software package for population genetics data analysis. Evolutionary Bioinformatics Online, 1: 47-50.

6. Fumihito, A., T. Miyake, S. Sumi, M. Takada, S. Ohno and N. Kondo. 1994. One subspecies of the red jungle fowl (Gallus gallus gallus) suffices as the matriarchic ancestor of all domestic breeds. National Academy of Sciences of the United States of America, 91: 12505-12509.

7. Guha, S., S.P. Goyal and V.K. Kashyap. 2006. Genomic variation in the mitochondrially encoded cytochorome $\mathrm{b}$ and 12s RNA genes. Characterization of eight endsngered pecorn species. Animal Genetics, 37: 262-265.

8. Hall, TA. 1999. BioEdit: a user-friendly biological sequence alignment editor and analysis program for windows 95/98/NT. Nucleic Acids Symposium Series, 4: 95-98.

9. Mohammadipestebik, F., N. Pirany, J. Shodja and A. Mohammadhashemi. 2011. Determination the mtDNA D-loop Sequence in Marandi Native Chicken Population and Its Phylogenic Relationships with Other Breeds. Research Journal of Animal Science, 21: 1-9 (In Persian).

10. Norollahi, H. and M.A. Kamali. 2011. A survey on performance of native poultry in rural areas of Fars. Animal Sciences Journal (Pajouhesh \& Sazandegi), 95: 8-12 (In Persian).

11. Oka, T., Y. Ino, K. Nomura, S. Kawashima, T. Kuwayama, H. Hanada, T. Amano, M. Takada, N. Takahata, Y. Hayashi and F. Akishinonomi-ya. 2007. Analysis of mtDNA sequences shows Japanese native chickens have multiple origins. Animal Genetics, 38: 287-293.

12. Pirany, N., A. Mohammadhashemi, S. Alijani, R. Rezazadeh Goli and S. Ghanbari. 2010. Molecular Analysis of Mazandrani native chicken population based on HVR-I region of Mitochondrial DNA Journal of Agricultural Biotechnology, 1: 53-65 (In Persian).

13. Silva, P., X. Guan, O. Ho-Shing, J. Jones, J. Xu, D. Hui, D. Notter and Smith E. 2008. Mitochondrial DNA-based analysis of genetic variation and relatedness among Sri Lankan indigenous chickens and the Ceylon jungle fowl (Gallus lafayetti). Animal Genetics, 40: 1-9.

14. Sultana, S. and H. Mannen. 2004. Polymorphism and evolutionary profile of mitochondrial DNA control region inferred from the sequences of Pakistani goats. Animal Science Journal, 75: 303-309.

15. Tamura, K., J. Dudley, M. Nei and S. Kumar. 2007. MEGA4: Molecular Evolutionary Genetics Analysis (MEGA) software version 4.0. Molecular Biology and Evolution, 24: 1596-1599.

16. Wallce, D.C. 1992. Mitochondrial genetics a paradigm for aging and degenerative diseases. Science, 256: 628-632.

17. Yacoub, H.A. and M.M. Fathi. 2013. Phylogenetic analysis using D-loop marker of mtDNA of Saudi native chicken strains. Animal Science Journal, 44: 5-6. 


\title{
Analysis of Genetic Diversity in Fars Native Chicken Based on Partial Mitochondrial DNA D-loop Region Sequences
}

\author{
Mahsa Nikoubin Borujeni ${ }^{1}$, Nasrollah Pirany ${ }^{2}$ and Fariba Rafiei Boroujeni ${ }^{3}$ \\ 1 and 3- M.Sc. Student and Assistant Professor, University of Shahrekord. \\ 2- Associate Professor, University of Shahrekord, \\ (Corresponding Author: napirany@gmail.com) \\ Received: March 10, 2014 \\ Accepted: January 10, 2015
}

\begin{abstract}
Native chickens are considered as national genetic resources and their conservation is very important from biodiversity aspects. The study of mitochondrial genome in one breed and comparing it with others can be a useful index for genetic diversity in that population. This study carried out for determining the sequences of mitochondrial high variable 1 (HVR-I) of D-loop region in Fars native chicken. Blood samples were collected randomly from 20 birds. After extracting DNA from whole blood, the HVR-I region was amplified using specific primers and then was sequenced. Totally, 12 sequences were obtained properly. After analyzing of the sequences, three haplotypes were identified with 5 single nucleotide polymorphic sites (SNP). These changes were mainly observed from nucleotides 217 to 446. After obtaining similar mtDNA sequence from GenBank, phylogenetic tree was drawn. Phylogenetic results indicated the Fars native chickens were clustered with Azerbaijan native, White Leghorn, Silky, Sonneratii, Barred Plymouth Rock, Iranian Marandi and Mazandarani native chickens. Therefore, we conclude that the Fars chicken might has some genetic similarities with these chicken breeds.
\end{abstract}

Keywords: Mitochondrial Genome, Highly variable region I (HVR-I), Single Nucleotide Polymorphism (SNP), Phylogenetic 